\title{
VAdriaC-IE Regimen
}

National Cancer Institute

\section{Source}

National Cancer Institute. VAdriaC-IE Regimen. NCI Thesaurus. Code C67232.

A regimen consisting of vincristine, doxorubicin and cyclophosphamide, alternating with ifosfamide and etoposide that can be used for the treatment of Ewing sarcoma and mesenchymal chondrosarcoma. 